\title{
Impact of the COVID-19 pandemic on emergency department CT for suspected diverticulitis: A natural experiment to explain patients' and clinicians' assessment of risk and willingness to undergo CT scanning?
}

Averi L. Gibson MD PhD ( $\triangle$ Averi.Gibson@umassmemorial.org )

University of Massachusetts Medical School https://orcid.org/0000-0003-2118-5430

Byron Y. Chen MD

University of Massachusetts Medical School

Max P. Rosen MD MPH

University of Massachusetts Medical School

S. Nicolas Paez MD

University of Massachusetts Medical School

Hao S. Lo MD MBA

University of Massachusetts Medical School

\section{Research Article}

Keywords: COVID-19, CT Utilization, Abdominopelvic CT, Diverticulitis.

Posted Date: September 11th, 2020

DOI: https://doi.org/10.21203/rs.3.rs-71883/v1

License: (c) (i) This work is licensed under a Creative Commons Attribution 4.0 International License.

Read Full License 


\section{Abstract}

Purpose: This study examined the impact of the COVID-19 pandemic on emergency department CT use for acute non-traumatic abdominal pain, to better understand why imaging volume so drastically decreased during the COVID-19 pandemic.

Methods: This was a retrospective review of emergency imaging volumes from January 5 to May 30, 2020. Weekly volume data were collected for total imaging studies, abdominopelvic CT, and abdominopelvic CTs positive for common causes of acute non-traumatic abdominal pain. Two emergency radiology attendings scored all diverticulitis cases independently and weekly volume data for uncomplicated and complicated diverticulitis cases was also collected. Volume data prior to and during the COVID-19 pandemic was compared, using 2019 volumes as a control.

Results: During the COVID-19 pandemic, overall emergency imaging volume decreased $30 \%$ compared to $2019(p=0.002)$. While the number of emergency abdominopelvic CTs positive for appendicitis and small bowel obstruction did not significantly change during the COVID-19 pandemic, the number of cases of diverticulitis decreased significantly compared to $2019(p=0.001)$. This reduction can be specifically attributed to decreased uncomplicated diverticulitis cases, as the number of uncomplicated diverticulitis cases dropped significantly $(p=0.002)$ while there was no significant difference in the number of complicated diverticulitis cases $(p=0.09)$.

Conclusions: Reduced emergency abdominopelvic CT volume during the COVID-19 pandemic can partially be explained by decreased imaging of lower acuity patients. This data may help formulate future strategies for imaging resource utilization with an improved understanding of the relationship between perceived imaging risk and symptom acuity.

\section{Introduction}

Since the first reported case from Wuhan, China in December 2019, coronavirus disease 2019 (COVID-19) has spread quickly across the world. To date, there have been over $23,000,000$ cases and over 810,000 deaths reported to the World Health Organization [1]. As of late August, the United States has been particularly affected, accounting for over 5,600,000 infections and 175,00 deaths [2]. Within this short time frame, the COVID-19 pandemic has dramatically impacted most aspects of the United States healthcare system. Within radiology, most nonurgent outpatient imaging was postponed.

Imaging volumes during the initial stages of the COVID-19 pandemic decreased across all patient care settings: inpatient, outpatient and emergency [3-6]. Studies have demonstrated that emergency department (ED) visits have also dramatically decreased during this time period [7]. Alarmingly, data suggest that some patients with critical conditions such as stroke or myocardial infarction may have avoided seeking emergency care [8-10]. Theories for explaining this phenomenon include patients fearing presentation to the hospital, self-medication of perceived minor symptoms, or no longer using the ED for chronic issues. However, there is a paucity of convincing data to explain this trend. 
XXXX has been one of several COVID-19 epicenters within the United States. The first COVID-19 case was reported in XXXXX on February 1, 2020 [11]. In response to rising case numbers, the state enacted a statewide stay at home advisory on March 24, 2020 [12]. The first case in XXXX County (home of the study institution) was reported on March 9, 2020 [11]. Afterwards the county followed a similar trend to the rest of the state, with cases peaking in late April/early May. After case numbers had fallen significantly, XXXXX lifted the stay at home advisory on May 19, 2020 and a slow, phased approach to reopening has begun [12].

The purpose of this study was to examine the impact of the COVID-19 pandemic on the ED use of computed tomography (CT) for acute non-traumatic abdominal pain, in order to better understand why CT volume in the ED has been so drastically impacted by the COVID-19 pandemic.

\section{Materials And Methods}

This was a retrospective review of imaging case volumes in one large academic healthcare system from January 6 to June 1, 2019 and January 5 to May 30,2020. An IRB waiver was obtained from the XXXX IRB for this study. The XXX Healthcare System is the largest health system in central XXXX, with an imaging volume of approximately 1 million studies in 2019. It encompasses five emergency departments in XXX County, including two urban campuses in XXXX and three community hospitals in the surrounding region. CT examinations from all five emergency departments were included in this study.

mPower Clinical Analytics (Nuance Communications, Inc, Burlington, MA) was used to aggregate ED imaging volume data for January 6 to June 1, 2019 and January 5 to May 30,2020. Weekly volume data for total imaging studies and abdominopelvic CT scans was collected. Aggregate weekly data was also collected for the number of adult abdominopelvic CT scans that were interpreted as positive for several common causes of acute non-traumatic abdominal pain, including appendicitis, diverticulitis, and small bowel obstruction, by emergency radiology attendings. The weekly number of whole-body trauma CT scans was also tracked over the same time period as an internal control.

\section{Imaging Analysis}

All ED abdominopelvic CT scans interpreted as positive for diverticulitis in patients 18 years and older during the defined study time frame were collected. These cases were scored independently by two emergency radiology attendings (X.X. with 8 years of experience and X.X. with 9 years of experience), who were blinded to the original CT interpretation. A score of 1 indicated uncomplicated diverticulitis, a score of 2 was complicated diverticulitis with pericolonic air bubbles, a score of 3 indicated complicated diverticulitis with an abscess, and a score of 4 was complicated diverticulitis with free air or fluid. A score of 0 was used when the study was interpreted as not consistent with diverticulitis. This scoring system reflects prior published methodology [13].

Both reviewers scored a total of 511 cases. 10 (1.95\%) cases had scores which differed between the two reviewers. For discordant scores, a single consensus score was agreed upon between the reviewers. 


\section{Statistical Analysis}

For all aggregate weekly data, the corresponding weeks in 2019 and 2020 were compared to allow for seasonal variation, with a week defined as Sunday to Saturday. Data from the 2020 time period prior to the XXXX stay at home advisory, (weeks 1 - 11), was compared to 2019 data from the same weeks, which served as a pre-COVID control, in order to identify any differences in imaging utilization that were present prior to the COVID pandemic. The COVID pandemic time period was then defined as the full duration of the XXXX stay at home advisory, weeks $12-19$.

All comparisons were performed using a student's t-test. A p-value of less than 0.05 was considered significant. The Cohen's kappa coefficient was calculated to assess agreement in diverticulitis case interpretation between the two radiologists. The Cohen's kappa coefficient was calculated using GraphPad software. All other statistics were performed using Microsoft Excel (version 14.7.2).

\section{Results}

We first evaluated overall ED imaging volume and the volume of emergency abdominopelvic CT scans, comparing 2019 to 2020 . Figure 1 displays the weekly trend for total ED imaging studies. Table 1 demonstrates the average weekly number of imaging studies prior to and during the COVID-19 pandemic, compared to the same time period in 2019.

In 2020, during the months prior to the COVID-19 pandemic, the total emergency imaging volume was significantly increased compared to the same time period in 2019. 2019 average weekly study volume was $2,544.2$ and 2020 average weekly study volume was $2,797.8(p=0.04)$. During the COVID-19 pandemic, the number of emergency imaging studies decreased by over $30 \%$ compared to the same time period in 2019, with a 2019 average weekly case volume of 2,390.8 and a 2020 average weekly case volume of 1,660.3 ( $p=0.002)$. The number of emergency abdominopelvic CTs followed a similar trend. There was no significant difference in the number of emergency abdominopelvic CTs performed during the months prior to the COVID-19 pandemic compared to the same time period in 2019. There was greater than $40 \%$ decrease in emergency abdominopelvic CTs during the COVID-19 pandemic compared to the same time period in 2019, with an average weekly volume of 319.8 CTs in 2019 and 191.4 CTs in 2020 (p $<0.001)$.

To better understand the drastic reduction in ED imaging volume during the COVID-19 pandemic, we determined the weekly number of CT scans that were interpreted as positive for several common causes of acute non-traumatic abdominal pain: appendicitis, diverticulitis, and small bowel obstruction. These diagnoses were tracked for several months prior to and during the COVID-19 pandemic and compared to volumes from the same time period in 2019. Figure 2 shows the trend over time for those diagnoses and Table 2 displays the average weekly number of diagnoses prior to and during the COVID-19 pandemic, compared to the same time period in 2019. As an internal control, the weekly number of ED trauma CT scans was also assessed (Supplementary Figure 1). 
There were no significant differences in the number of cases of appendicitis or small bowel obstruction, when comparing 2019 and 2020, either prior to or during the COVID-19 pandemic. However, the average weekly number of positive diverticulitis cases dramatically decreased during the COVID-19 weeks. In the months prior to the pandemic, the average weekly number of diverticulitis cases was not significantly different between 2019 and 2020, 13.8 in 2019 and 12.6 in 2020 ( $p=0.43$ ). However, during the COVID-19 pandemic, the average weekly number of positive diverticulitis cases decreased to 5.1 , compared to 13.5 during the same time period in $2019(p=0.001)$. The weekly number of diverticulitis cases began to increase at the end of the pandemic "first wave" and became equivalent to the same period in 2019 by the end of the study period, suggesting that this was a transient reduction.

The decrease specifically in diverticulitis cases raised the concern that patients may have delayed presentation to the ED, which may have in turn increased disease severity at the time of presentation. To determine if the severity of diverticulitis cases presenting to the ED for care during the COVID-19 pandemic had changed, all ED CT scans positive for diverticulitis during the study period in 2019 and 2020 were scored independently by two emergency radiology attendings for disease severity at the time of presentation. The scoring system delineated between uncomplicated and complicated cases of diverticulitis. For the complicated cases, the severity of complication was also graded.

A total of 511 cases were scored, demonstrating high concordance between the two radiologists (kappa coefficient $=0.95$ ). Of the 511 total cases, 300 were from the 2019 study period and 211 were from the 2020 study period. Figure 3 demonstrates the weekly number of cases of uncomplicated and complicated diverticulitis over time. Table 3 shows the average weekly number of cases that were positive for uncomplicated and complicated diverticulitis prior to and during the COVID-19 pandemic, compared to the same time period in 2019.

We found that the dramatic reduction in overall ED diverticulitis cases during the COVID-19 pandemic can be attributed specifically to a large decrease in the number of uncomplicated diverticulitis cases. In the months prior to the pandemic, the average weekly number of uncomplicated diverticulitis cases was comparable to the same time period in 2019, 10.6 in 2019 and 9.6 in $2020(p=0.41)$. The average weekly number of complicated diverticulitis cases in the months prior to the pandemic was also comparable to the same time period in 2019, 3.0 in 2019 and 2.3 in 2020 ( $p=0.26)$. However, during the COVID-19 pandemic, the average weekly number of uncomplicated diverticulitis cases plunged to 3.6 , compared to 10.8 during the same time period in $2019(p=0.002)$. In contrast, the average weekly number of complicated diverticulitis cases was not significantly different from the same time period in 2019, 1.5 during the COVID-19 pandemic, compared to 2.4 in $2019(p=0.09)$.

\section{Discussion}

The COVID-19 pandemic has had a rapidly evolving impact on the U.S. healthcare system. Several studies have shown that ED imaging volume decreased substantially during the COVID-19 pandemic $[3,4,6]$. This contrasts with the pre-pandemic trend in ED imaging, particularly $C T$, which had been 
increasing for the past decade [14-16], as convincing data has shown that early CT diagnosis of certain conditions, such as appendicitis and uncomplicated diverticulitis, reduces hospital resource use and improves patient outcomes $[17,18]$. There are several potential reasons why ED CT volume decreased during the COVID-19 pandemic, as one would expect the incidence of diseases requiring an abdominopelvic CT in the ED should remain constant or increased. Our data demonstrates that patients with higher acuity conditions usually requiring surgery and/or hospitalization (e.g., appendicitis, small bowel obstruction, complicated diverticulitis) were still being diagnosed by CT at the same rate as before COVID-19. However, patients with uncomplicated diverticulitis, a lower acuity disease that can often be treated as an outpatient, were no longer presenting to the ED for CT scanning.

This paradigm may be explained by understanding the history of abdominal CT utilization in the ED to diagnose diverticulitis. In 1984, Hulnick et al. published a series of 43 consecutive patients imaged with CT at New York University (NYU) Medical Center between 1979 and 1983 [19]. Following this publication, Ferris Hall, writing in an editorial in Radiology stated " ... no evidence is provided showing that CT scans alter treatment, expedited or obviated surgery, shortened hospital stay or reduced complication" [20]. Almost 30 years later, Pandharipande et al documented that CT changed the diagnosis of $67 \%$ of patients who underwent CT in the ED for suspected diverticulitis [21].

Over this 30-year time frame, the speed, availability and resolution of CT increased and barriers to performing a CT in the ED steadily decreased. The number of patients scanned increased; and the "emergency" barium enema was eliminated from our diagnostic algorithms. However, with the onset of COVID-19, a new barrier to CT scanning was abruptly inserted into the risk/benefit calculation. Now patients and clinicians had to factor the risk of COVID-19 exposure in the ED and clinicians had to factor in the diversion of CT resources away from other COVID-19 related triage.

The fact that CT for uncomplicated diverticulitis decreased during COVID-19, but the use of CT for complicated diverticulitis, appendicitis, and small bowel obstruction was unchanged suggests an interaction between symptom acuity and perceived risks/barriers to imaging that patients and/or clinicians balance when deciding if imaging is necessary. The American Academy of Family Physicians (AAFP) recommendations for diagnosis and management of acute diverticulitis from 2013 state that "imaging is not necessary in most patients with mild symptoms" [22]. The ACR appropriateness criteria from 2019 agrees [23]. It may be that provider willingness to recommend a CT scan for mild symptoms dropped in the face of higher perceived imaging risk. It is also possible that health care providers were more comfortable empirically treating patients with a prior history of diverticulitis without imaging rather than risking COVID-19 exposure during an ED visit. And of course one must consider that patients with mild symptoms may have chosen not to seek care at all.

It is also important to note that while the number of abdominopelvic CTs positive for uncomplicated diverticulitis dropped significantly, there was not a corresponding increase in abdominopelvic CTs positive for complicated diverticulitis. This suggests that while patients with diverticulitis may have 
delayed presentation to the ED in the face of risks associated with COVID-19 exposure, this possible delay did not result in a change in disease severity at the time of presentation.

This study has several limitations. One is its retrospective design, allowing for correlational analysis only. A second limitation is the size of the study, which uses data from a single health system. Romero et al recently reported that during the COVID-19 pandemic, fewer patients presented with acute appendicitis to the ED, and those who did presented at a more severe stage [24]. Our data did not corroborate this conclusion. Possible explanations include small sample size, disparate patient populations and different clinical courses for appendicitis versus diverticulitis.

An additional limitation is that our timeframe for analysis starts with presentation to the ED. We do not know how many patients were triaged towards or away from the ED based on recommendations from their primary care providers or other providers. As discussed above, this may have had a large impact on

the number and severity of cases that did present to the ED. Finally, this study was designed assuming no interaction between diverticulitis incidence and COVID-19 infection. While there has been no data to suggest a causative relationship, COVID-19 is known to affect the gastrointestinal system as many patients do present with gastrointestinal symptoms such as nausea, vomiting or diarrhea [25].

In conclusion, our data suggest that the decrease in abdominopelvic CT scans during the COVID-19 pandemic can be at least partially attributed to a decrease in imaging of lower acuity patients. Our data support the claim that patients with higher acuity abdominal pain did eventually undergo CT in the ED and that possible delayed presentation, at least for patients with diverticulitis, did not result in an increase in disease severity at the time of presentation. These results help explain the marked reduction in the use of $C T$ in the ED during the COVID-19 pandemic and may help to define future strategies for imaging resources utilization. For example, as health care systems and patients have rapidly expanded their use of telemedicine, it may be useful to consider the use of an ED telemedicine consult prior to ED presentation for patients with specific complaints who are concerned about coming the ED, in order to help triage lower risk patients to reduce exposure risk and decongest the ED in times of high resource use. In addition, health care providers' experience with reduced reliance on imaging during COVID may prompt renewed discussions about the most appropriate use of CT in the ED and may lead to empiric treatment of patients with suspected uncomplicated diverticulitis without first having patients undergo a CT scan.

\section{Declarations}

Funding There were no sources of funding for this work.

Conflicts of Interest/Competing Interests Dr. Rosen serves on the scientific advisory boards for Percetive Informatics, Lung Life Al, Canon Medical Systems. Drs. Gibson, Chen, Paex and Lo declare that they have no disclosures.

Ethics Approval This study was designated not human subjects research by the University of Massachusetts Medical School IRB on 6/15/2020. An IRB waiver was obtained. 
Consent to Participate This study was designed not human subjects research by the University of Massachusetts Medical School IRB, therefore informed consent was not necessary.

Consent for Publication This study was designed not human subjects research by the University of Massachusetts Medical School IRB, therefore consent for publication was not necessary.

Availability of Data and Material All of the data reported in this manuscript is available by request.

Code Availability Not applicable.

Author's Contributions All authors contributed significantly to the concept, content or design of this article. The article was written by Dr. Gibson and revised by Drs. Chen, Rosen, Paez and Lo. All authors approved of the final version of the manuscript. The authors declare that they had full access to all of the data in this study and the authors take complete responsibility for the integrity of the data and the accuracy of the data analysis.

\section{References}

1. WHO Coronavirus Disease (COVID-19) Dashboard. (2020). https://covid19.who.int/. Accessed Accessed August 24, 2020.

2. Coronavirus Disease 2019 (COVID-19): Cases in the U.S. (2020) Centers for Disease Control and Prevention (CDC). https://www.cdc.gov/coronavirus/2019-ncov/cases-updates/cases-in-us.html. Accessed Accessed August 24, 2020.

3. Naidich JJ, Boltyenkov A, Wang JJ, Chusid J, Hughes D, Sanelli PC (2020) Impact of the COVID-19 Pandemic on Imaging Case Volumes. J Am Coll Radiol. doi:10.1016/j.jacr.2020.05.004

4. Cavallo JJ, Forman HP (2020) The Economic Impact of the COVID-19 Pandemic on Radiology Practices. Radiology:201495. doi:10.1148/radiol.2020201495

5. Naidich JJ, Boltyenkov A, Wang JJ, Chusid J, Hughes D, Sanelli PC (2020) COVID-19 Pandemic Shifts Inpatient Imaging Utilization. J Am Coll Radiol. doi:10.1016/j.jacr.2020.06.011

6. O'Brien CM, Jung K, Dang W, Hyun-Jung J, Kielar AZ (2020) Collateral damage: the impact of the COVID-19 pandemic on acute abdominal emergency presentations. J Am Coll Radiol. doi: https://doi.org/10.1016/j.jacr.2020.08.010.

7. Hartnett K, Kite-Powell A, DeViews J, Coletta M, Boehmer T, Adjemian J, Gundlapalli A (2020) Impact of the COVID-19 Pandemic on Emergency Department Visits - United States, January 1, 2019-May 30, 2020. Centers for Disease Control and Prevention Morbidity and Mortality Weekly Report 69. doi:10.15585/mmwr.mm6923e1

8. Kansagra AP, Goyal MS, Hamilton S, Albers GW (2020) Collateral Effect of Covid-19 on Stroke Evaluation in the United States. N Engl J Med. doi:10.1056/NEJMc2014816

9. Garcia S, Albaghdadi MS, Meraj PM, Schmidt C, Garberich R, Jaffer FA, Dixon S, Rade JJ, Tannenbaum M, Chambers J, Huang PP, Henry TD (2020) Reduction in ST-Segment Elevation 
Cardiac Catheterization Laboratory Activations in the United States During COVID-19 Pandemic. J Am Coll Cardiol 75 (22):2871-2872. doi:10.1016/j.jacc.2020.04.011

10. Lange S, Ritchey M, Goodman A, Dias T, Twentyman E, Fuld J, Schieve L, Imperatore G, Benoit S, KitePowell A, Stein Z, Peacock G, Dowling N, Briss P, Hacker K, Gundlapalli A, Yang Q (2020) Potential Indirect Effects of the COVID-19 Pandemic on Use of Emergency Departments for Acute LifeThreatening Conditions - United States, January - May 2020. Centers for Disease Control and Prevention Morbidity and Mortality Weekly Report 69. doi:10.15585/mmwr.mm6925e2

11. Archive of COVID-19 cases in Massachusetts. (2020) Massachusetts Department of Public Health. https://www.mass.gov/info-details/archive-of-covid-19-cases-in-massachusetts. Accessed Accessed June 17, 2020. 2020

12. Coronavirus disease (COVID-19) Timeline. (2020) Boston Public Health Commission. https://www.boston.gov/departments/public-health-commission/coronavirus-timeline. Accessed Accessed June 17, 2020.

13. Sartelli M, Moore FA, Ansaloni L, Di Saverio S, Coccolini F, Griffiths EA, Coimbra R, Agresta F, Sakakushev B, Ordonez CA, Abu-Zidan FM, Karamarkovic A, Augustin G, Costa Navarro D, Ulrych J, Demetrashvili Z, Melo RB, Marwah S, Zachariah SK, Wani I, Shelat VG, Kim JI, McFarlane M, Pintar T, Rems M, Bala M, Ben-Ishay O, Gomes CA, Faro MP, Pereira GA, Jr., Catani M, Baiocchi G, Bini R, Anania G, Negoi I, Kecbaja Z, Omari AH, Cui Y, Kenig J, Sato N, Vereczkei A, Skrovina M, Das K, Bellanova G, Di Carlo I, Segovia Lohse HA, Kong V, Kok KY, Massalou D, Smirnov D, Gachabayov M, Gkiokas G, Marinis A, Spyropoulos C, Nikolopoulos I, Bouliaris K, Tepp J, Lohsiriwat V, Colak E, Isik A, Rios-Cruz D, Soto R, Abbas A, Trana C, Caproli E, Soldatenkova D, Corcione F, Piazza D, Catena F (2015) A proposal for a CT driven classification of left colon acute diverticulitis. World J Emerg Surg 10:3. doi:10.1186/1749-7922-10-3

14. Selvarajan SK, Levin DC, Parker L (2019) The Increasing Use of Emergency Department Imaging in the United States: Is It Appropriate? AJR Am J Roentgenol 213 (4):W180-W184. doi:10.2214/AJR.19.21386

15. Bellolio MF, Heien HC, Sangaralingham LR, Jeffery MM, Campbell RL, Cabrera D, Shah ND, Hess EP (2017) Increased Computed Tomography Utilization in the Emergency Department and Its Association with Hospital Admission. West J Emerg Med 18 (5):835-845. doi:10.5811/westjem.2017.5.34152

16. Pola A, Corbella D, Righini A, Torresin A, Colombo PE, Vismara L, Trombetta L, Maddalo M, Introini MV, Tinelli D, Strohmenger L, Garattini G, Munari A, Triulzi F (2018) Computed tomography use in a large Italian region: trend analysis 2004-2014 of emergency and outpatient CT examinations in children and adults. Eur Radiol 28 (6):2308-2318. doi:10.1007/s00330-017-5225-x

17. Rao PM, Rhea JT, Novelline RA, Mostafavi AA, McCabe CJ (1998) Effect of computed tomography of the appendix on treatment of patients and use of hospital resources. N Engl J Med 338 (3):141-146. doi:10.1056/NEJM199801153380301 
18. Juszczyk K, Ireland K, Thomas B, Kroon HM, Hollington P (2019) Reduction in hospital admissions with an early computed tomography scan: results of an outpatient management protocol for uncomplicated acute diverticulitis. ANZ J Surg 89 (9):1085-1090. doi:10.1111/ans. 15285

19. Hulnick DH, Megibow AJ, Balthazar EJ, Naidich DP, Bosniak MA (1984) Computed tomography in the evaluation of diverticulitis. Radiology 152 (2):491-495. doi:10.1148/radiology.152.2.6739821

20. Hall FM (1985) Computed tomography in the evaluation of diverticulitis. Radiology 154 (3):835-836. doi:10.1148/radiology.154.3.835-c

21. Pandharipande PV, Reisner AT, Binder WD, Zaheer A, Gunn ML, Linnau KF, Miller CM, Avery LL, Herring MS, Tramontano AC, Dowling EC, Abujudeh HH, Eisenberg JD, Halpern EF, Donelan K, Gazelle GS (2016) CT in the Emergency Department: A Real-Time Study of Changes in Physician Decision Making. Radiology 278 (3):812-821. doi:10.1148/radiol.2015150473

22. Wilkins T, Embry K, George R (2013) Diagnosis and management of acute diverticulitis. Am Fam Physician 87 (9):612-620

23. Expert Panel on Gastrointestinal I, Galgano SJ, McNamara MM, Peterson CM, Kim DH, Fowler KJ, Camacho MA, Cash BD, Chang KJ, Feig BW, Gage KL, Garcia EM, Kambadakone AR, Levy AD, Liu PS, Marin D, Moreno C, Pietryga JA, Smith MP, Weinstein S, Carucci LR (2019) ACR Appropriateness Criteria((R)) Left Lower Quadrant Pain-Suspected Diverticulitis. J Am Coll Radiol 16 (5S):S141-S149. doi:10.1016/j.jacr.2019.02.015

24. Romero J, Valencia S, Guerrero A (2020) Acute Appendicitis During Coronavirus Disease 2019 (COVID-19): Changes in Clinical Presentation and CT Findings. J Am Coll Radiol. doi:10.1016/j.jacr.2020.06.002

25. Symptoms of Coronavirus (2020) Centers for Disease Control and Prevention. https://www.cdc.gov/coronavirus/2019-ncov/symptoms-testing/symptoms.html. Accessed June 30, 2020

\section{Figures}


A

Number of Total ED Imaging Studies by Week

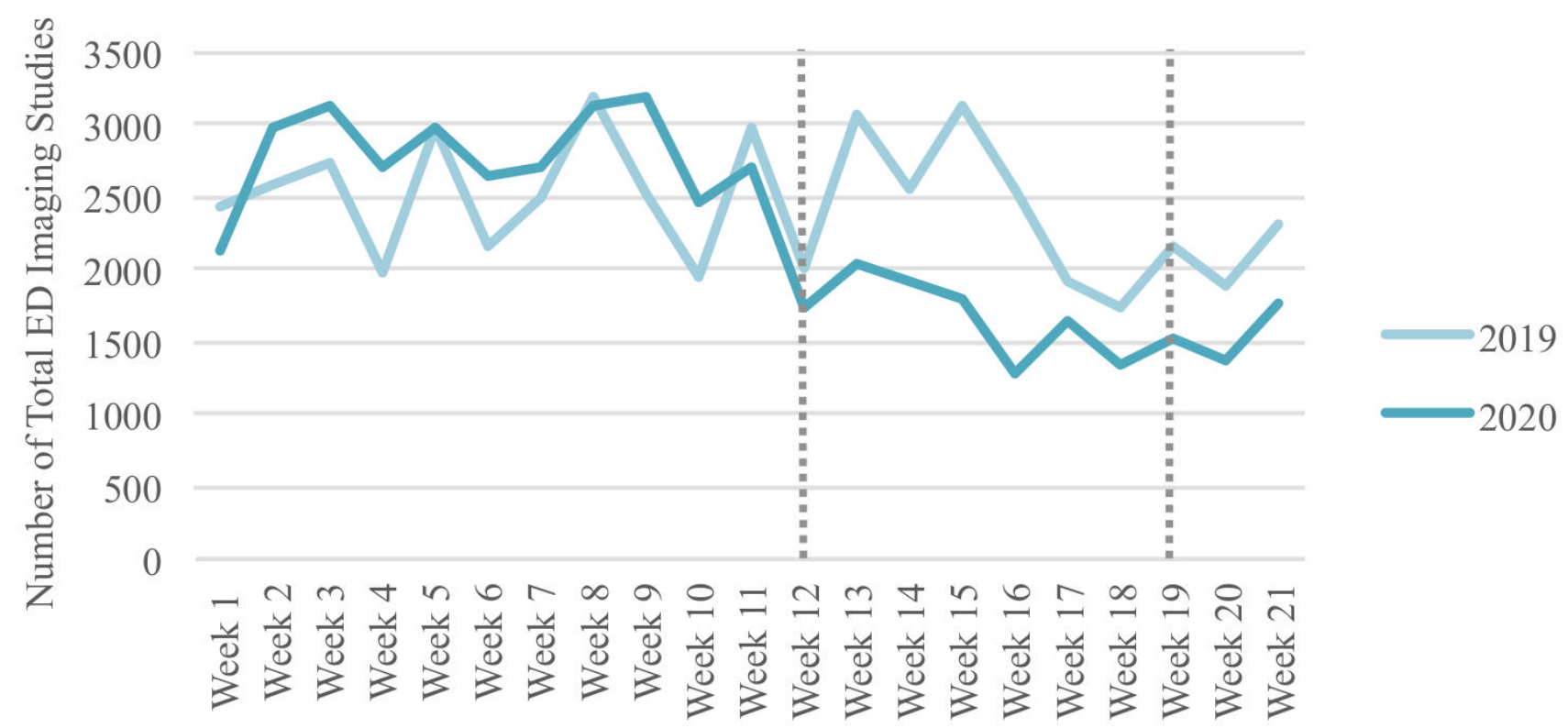

B

\section{Number of ED Abdominal CTs by Week}

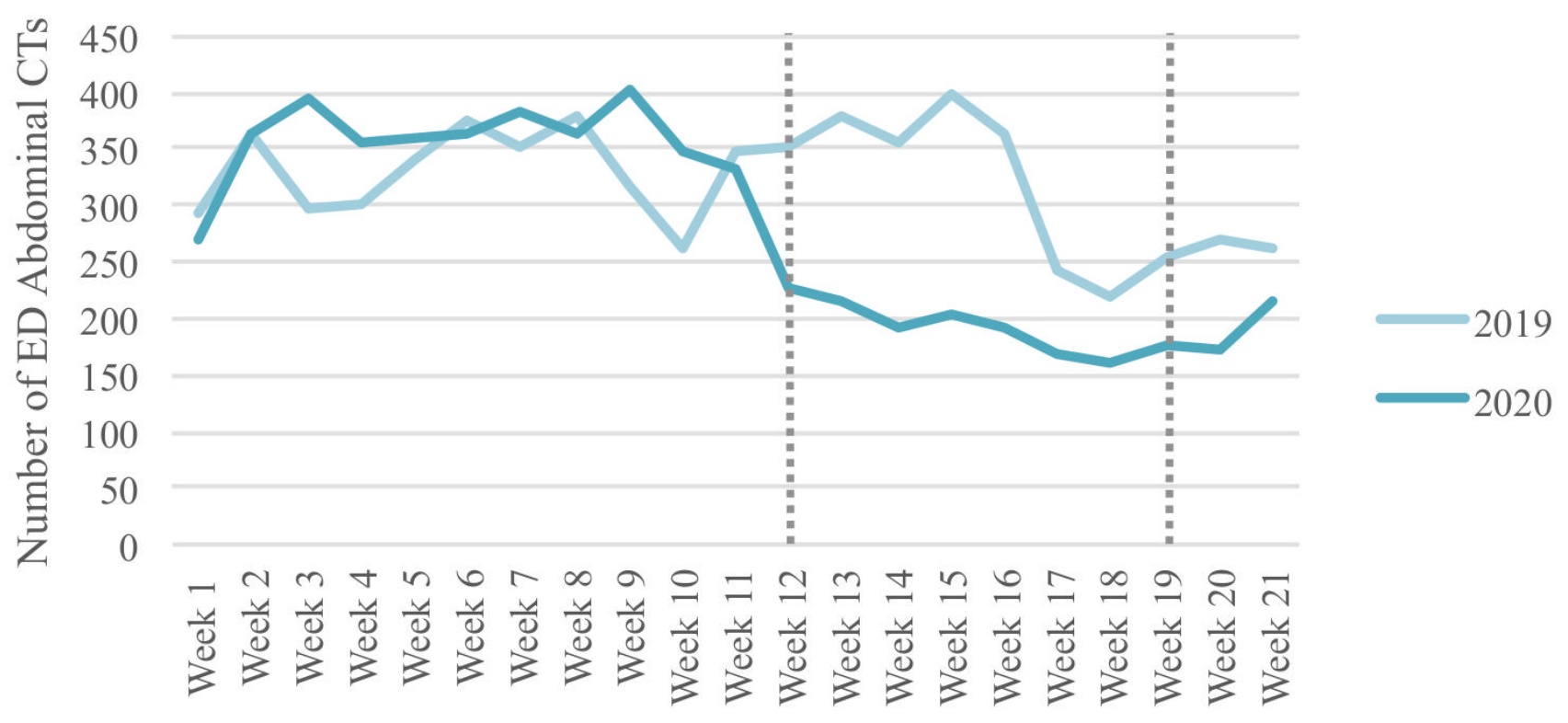

\section{Figure 1}

The trend over time in A. total ED imaging volume and B. ED abdomen/pelvis CTs by week during the preCOVID-19 and COVID-19 pandemic weeks in 2020, compared to the same time period in 2019. The first vertical gray line denotes the beginning of the Massachusetts stay at home advisory and the second vertical gray line denotes the end of the stay at home advisory. 
A

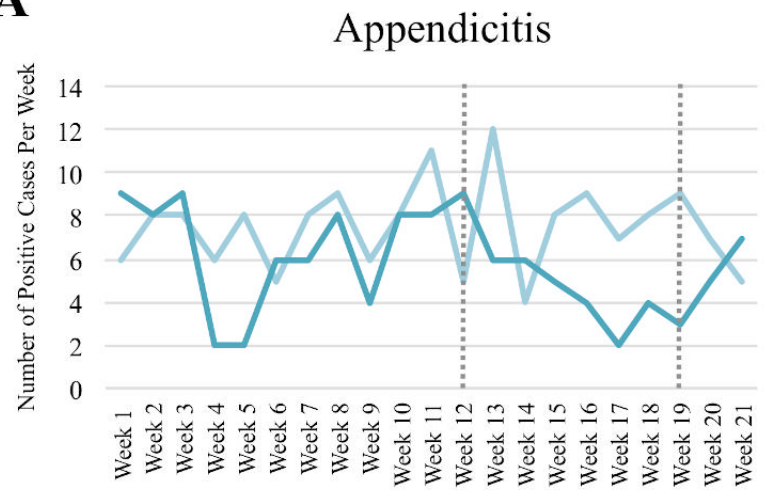

C

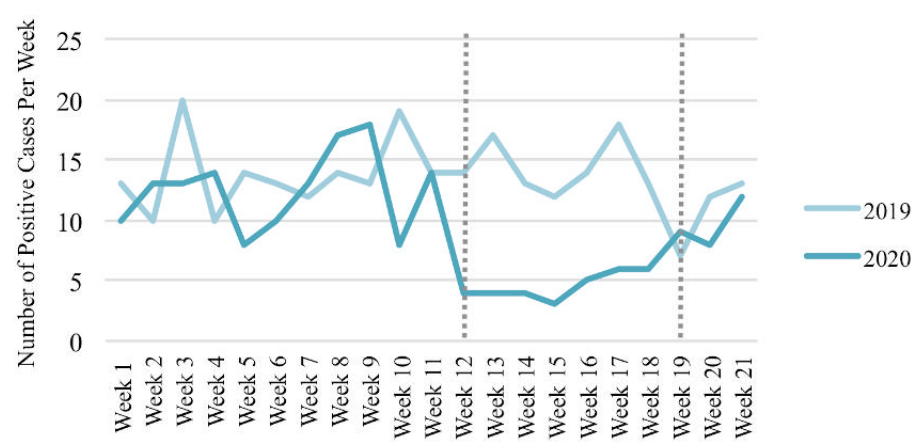

B Small Bowel Obstruction

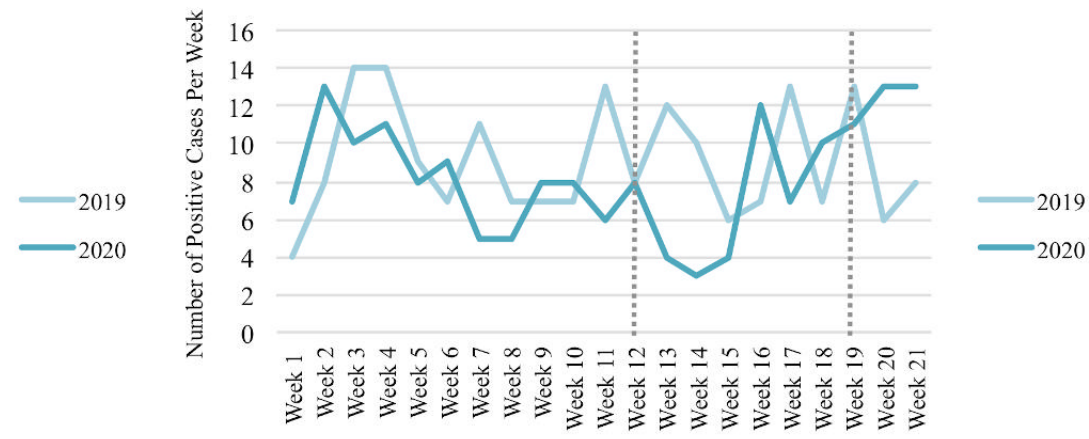

\section{Figure 2}

The trend over time in ED CT cases positive for specific abdominal pathology by week during the preCOVID-19 and COVID-19 pandemic weeks in 2020, compared to the same time period in 2019. The first vertical gray line denotes the beginning of the Massachusetts stay at home advisory and the second vertical gray line denotes the end of the stay at home advisory. 
A

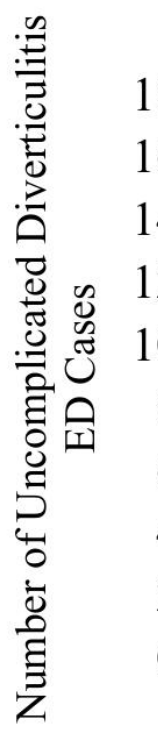

\section{Uncomplicated Diverticulitis Cases by Week}

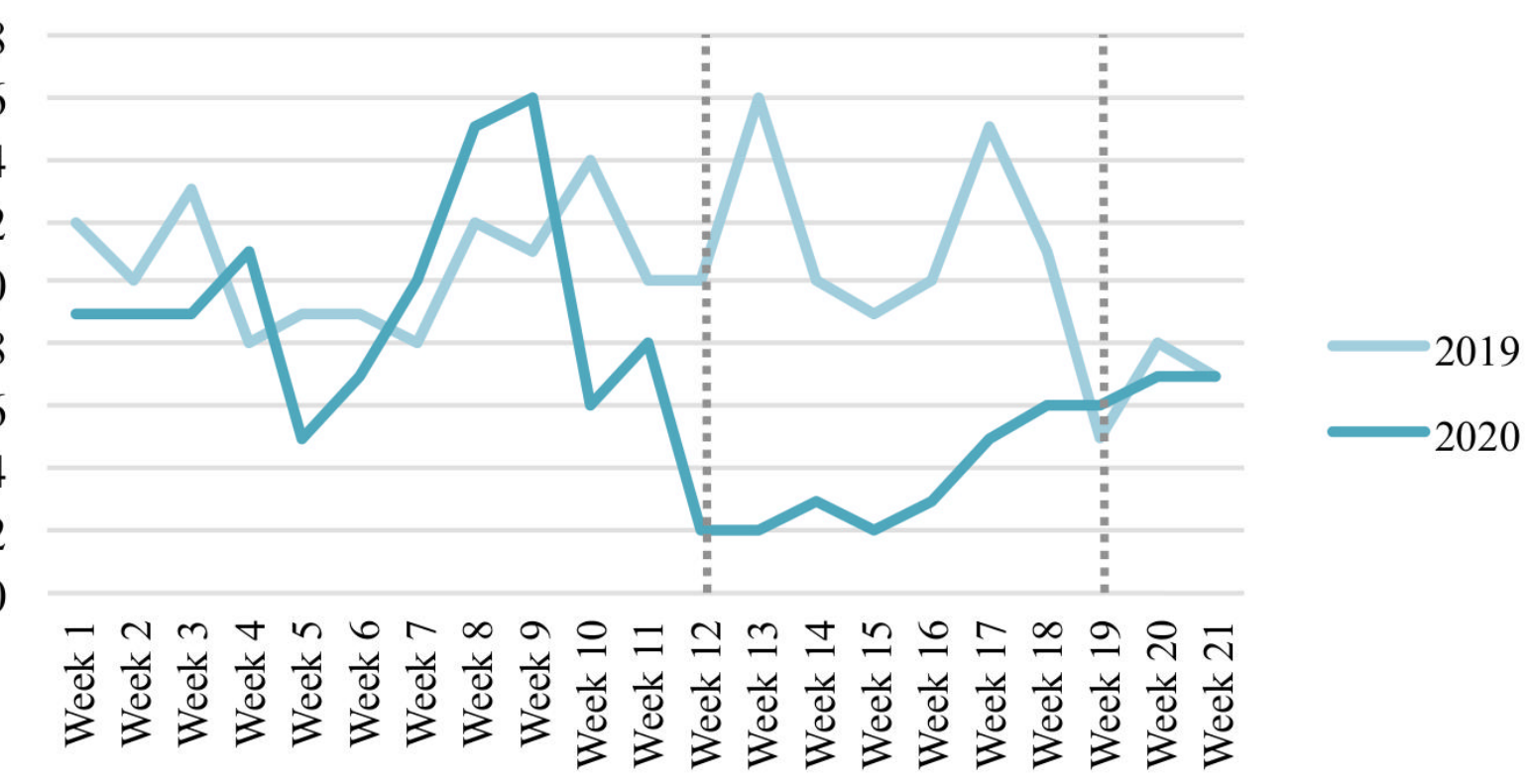

\section{Complicated Diverticulitis Cases by Week}

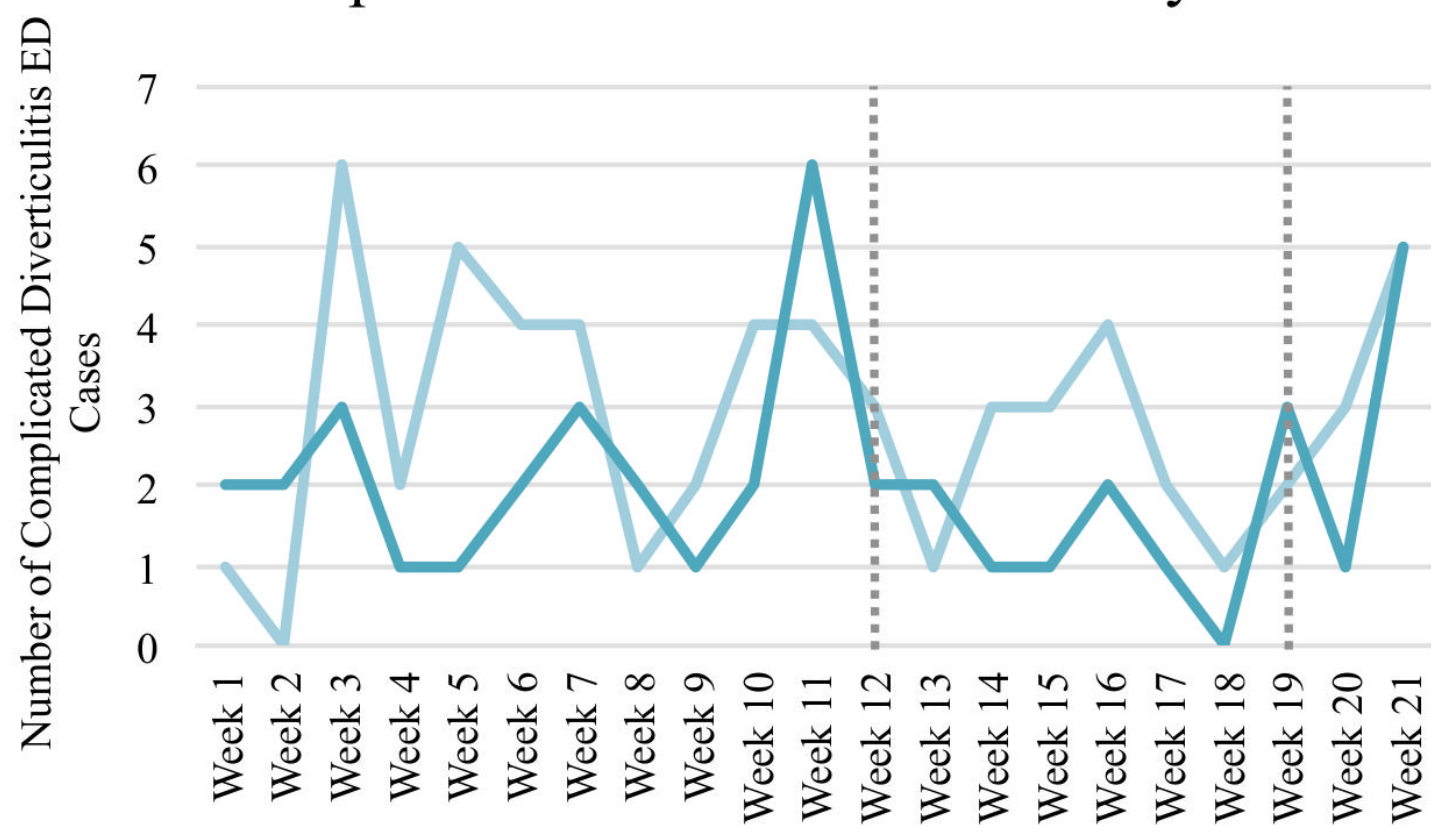

Figure 3

The trend over time in ED CT cases positive for A. uncomplicated and B. complicated diverticulitis by week during the pre-COVID-19 and COVID-19 pandemic weeks in 2020, compared to the same time period in 2019. The first vertical gray line denotes the beginning of the Massachusetts stay at home advisory and the second vertical gray line denotes the end of the stay at home advisory. 


\section{Supplementary Files}

This is a list of supplementary files associated with this preprint. Click to download.

- FigS1.tif 